\title{
Anatomic relationship of the proximal nail matrix to the extensor hallucis longus tendon insertion
}

\author{
P. Palomo López, ${ }^{1}$ R. Becerro de Bengoa Vallejo, ${ }^{2}$ D. López López, ${ }^{4}$ J.C. Prados Frutos, ${ }^{3}$ \\ J. Alfonso Murillo González, ${ }^{5}$ M.E. Losa Iglesias ${ }^{3, *}$ \\ ${ }^{1}$ Centro Universitario de Plasencia, Universidad de Extremadura, Plasencia, Spain \\ ${ }^{2}$ Facultad de Enfermería, Fisioterapia y Podologia, Universidad Complutense de Madrid, Madrid, Spain \\ ${ }^{3}$ Facultad Ciencias de la Salud, Universidad Rey Juan Carlos, Madrid, Spain \\ ${ }^{4}$ Departamento de Ciencias de la Salud, Universidad de A Coruña, Ferrol, Spain \\ ${ }^{5}$ Departamento Anatomía y Embriología Humana I. Facultad de Medicina, Universidad Complutense de Madrid, Madrid, Spain \\ *Correspondence: M. E. Losa Iglesias. E-mail: marta.losa@urjc.es
}

\section{Abstract}

Background The purpose of this study was to delineate the relationship of the terminal extensor hallucis longus tendon insertion to the proximal limit of the nail matrix of the great toe.

Material and methods Fifty fresh-frozen human cadaver great toes with no evidence of trauma (average age, 62.5 years; 29 males and 21 females) were used for this study. Under 25X magnification, the proximal limit of the nail matrix and the terminal bony insertion of the extensor hallucis longus tendons were identified. The distance from the terminal tendon insertion to the nail matrix was ascertained using precision calipers, an optical microscope, and AUTOCAD ${ }^{\circledR}$ software for windows. Twenty-five great toes were placed in a neutral formalin solution and further analysed by histological longitudinal-sections. The specimens were stained with haematoxylin and eosin and examined microscopically to determine the presence of the extensor hallucis longus tendon along the dorsal aspect of the distal phalanx of each great toe.

Results The main result we found in great toes was that the extensor tendon is between the matrix and the phalanx and extends dorsally to the distal aspect of the distal phalanx in all, $100 \%$, specimens. The nail matrix of the great toe is not attached to the periosteum of the dorsal aspect of the base of the distal phalanx as is the case for fingers, because the extensor hallucis tendon is plantar or directly underneath the nail matrix and the tendon is dorsal to the bone.

Conclusions We have found that the extensor tendon is between the matrix and the phalanx and extends dorsally to the distal aspect of the distal phalanx. The nail matrix of the great toe is not attached to the periosteum of the dorsal aspect of the base of distal phalanx as is the case in fingers, because the extensor hallucis tendon is plantar or directly underneath the nail matrix and the tendon is dorsal to the bone. Our anatomic study demonstrates that the proximal limit of the matrix and nail bed of the human great toe are dorsal and overlapping the terminal extensor hallucis longus tendon until its distal bony insertion in all specimens.

Received: 5 December 2014; Accepted: 18 February 2015

\section{Conflicts of interest}

None declared.

\section{Funding sources}

None declared.

\section{Introduction}

The great toe is the most commonly injured part of the foot. These injuries most often involve the great toe-tip, the nail matrix and the nail plate. ${ }^{1,2}$ Surgery of the distal phalanx, nail matrix and nail plate of the great toe is often required, not only to address injuries secondary to trauma but also to treat a variety of problems, including infections, tumours, iatrogenic injury, degenerative conditions and self-inflicted injury. ${ }^{3,4}$ Moreover, nail bed reconstruction requires an understanding of the anatomy of the nail matrix and the intimate relationship between the nail and its surrounding tissues.

Clinical experience has demonstrated the vulnerability of the matrix to injury and subsequently to deformity after surgical exposure of the dorsal aspect of the great toe and of the nail 
plate. The matrix is the sole source of the nail plate. Compared with the distal nail bed it has a much higher potential for scarring. ${ }^{5}$ Nail deformity is not infrequent after treatment of the aforementioned conditions. ${ }^{6}$

Knowledge of the proximal limit of the matrix is essential during operative exposure to avoid permanent nail deformity. In spite of the numerous studies examining the anatomy of the nail unit, ${ }^{6-14}$ to our knowledge, no study has quantified the anatomic relationship between the proximal nail matrix and the terminal extensor hallucis longus tendon insertion. Our study delineates the relationship between these two structures. The main goal of this study was to obtain measurements and study the anatomy of the terminal extensor hallucis longus and its adjacent structures.

\section{Materials and methods}

Fifty fresh-frozen human cadaver great toes with no evidence of trauma were used for this study. The average age was 62.5 years (range, 45-80 years). There were 29 men and 21 women. The great toes came from 24 right feet and 26 left feet. The human cadavers belonged to the Department of Human Anatomy and Embryology I of the Complutense University of Madrid's School of Medicine.

Twenty-five great toes were used for anatomical dissection and another 25 great toes were placed in a neutral formalin solution for histological analysis. We used standard longitudinal sections, each decalcified, processed and stained with haematoxylin and eosin followed by microscopic examination to determine the insertion or presence of extensor hallucis longus tendon along the dorsal aspect of the distal phalanx.

A longitudinal dorsal skin incision was made along the length of the middle and distal phalanges extending through the eponychium. Two radial incisions were also made proximal to the junctions of the nail fold and nail walls. ${ }^{15,16}$ The skin flaps were retracted to expose the dorsal part of the matrix and the entire length of the nail plate. The toenail was then removed atraumatically, preserving the matrix, nail bed, and eponychium. This was achieved by first loosening the nail plate from the matrix using a fine elevator.

The nail plate was then gently elevated and sharply excised from side to side. Removal of the nail plate enabled accurate inspection of the borders of the nail matrix.

The proximal limit of the nail matrix was identified by visual inspection and by noting the location of the proximal origin of the nail plate. The proximal origin of the nail plate constituted the proximal limit of the nail matrix. The nail matrix is white, the nail bed is red (Fig. 1).

The proximal limit of the nail matrix with respect to the distal limit of the terminal bony insertion of the extensor hallucis longus tendon was identified using both a $25 \mathrm{X}$ magnification optical microscope, and by visual inspection.

The insertion of the terminal extensor hallucis longus tendon was identified by visual inspection and confirmed by noting the

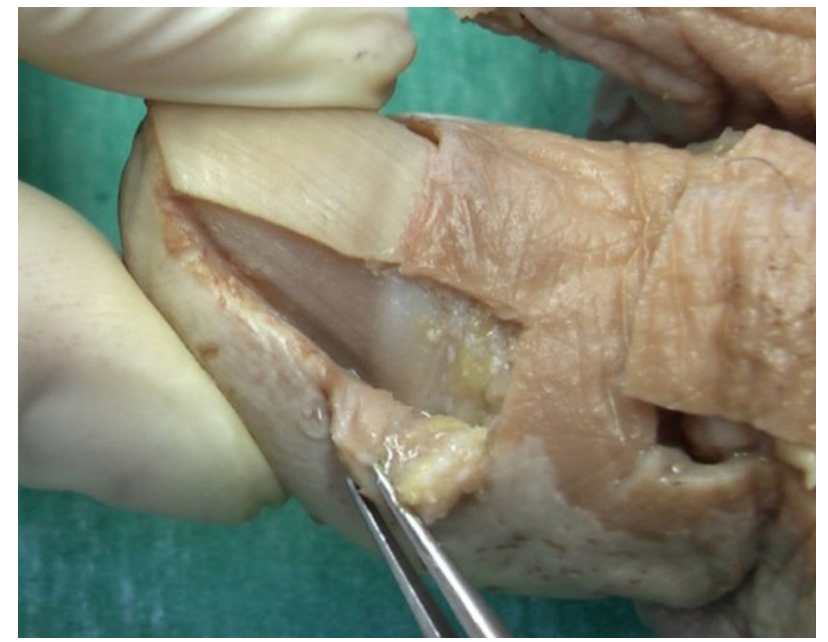

Figure 1 The nail plate, nail matrix, and nail bed change colour.

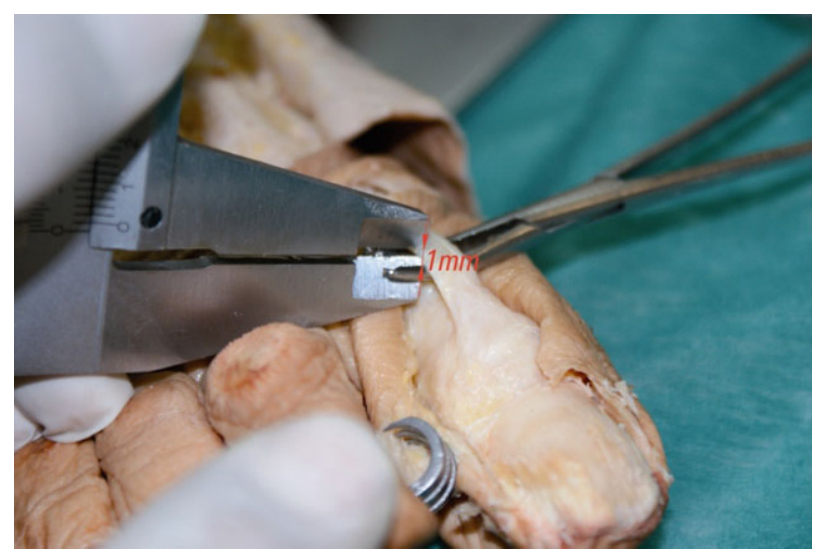

Figure 2 Exposing the nail matrix, the distal interphalangeal joint, and the terminal extensor hallucis longus tendon.

point at which a fine clamp could no longer be advanced distally underneath of the tendon (Fig. 2).

The tendon was released from the base of the distal phalanx using a no. 15 surgical blade, and the tendon was resected from the dorsal aspect of the distal phalanx bone until total desinsertion was completed as described by other authors. ${ }^{17}$ Each measurement was performed using digital precision calipers as described by other authors, ${ }^{18}$ with a resolution of $0.01 \mathrm{~mm}$ (VELDCA 150, Madrid, Spain). The measurements were made by placing each of the caliper tips on the proximal and distal ends for the length and on the mid-portion for the width of the measured structures.

The terminal extensor hallucis tendon was observed taking the distal interphalangeal joint (DIPJ) line as reference (Fig. 3). 


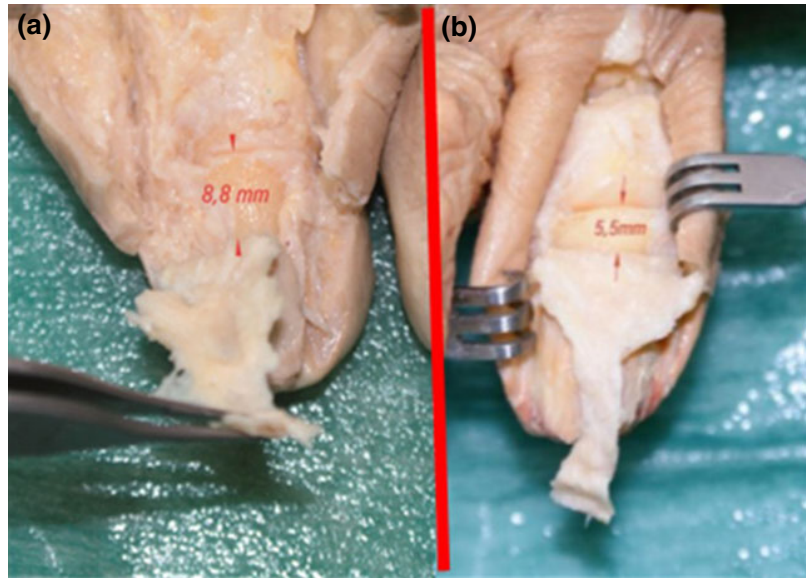

Figure 3 Exposing the distal insertion of the extensor hallucis longus tendon at the base of distal phalanx until proximal nail matrix fold is reached. In (a) the tendon attached to the dorsal aspect of the base of the distal phalanx is $8.8 \mathrm{~mm}$ from distal interphalangeal joint (DIPJ) line to the tendon reaching the proximal nail fold matrix (between small arrows) and (b), the same measurement in another specimen is $5.5 \mathrm{~mm}$.

The anatomical landmarks measured were numbered for reference from 1 to 8 .

Variable 1. The matrix width is measured from lateral to medial side at the most proximal portion of the great toe.

Variable 2. The same measurement was performed at the matrix but in its distal aspect, where colour changes with the nail bed can be seen.

Variable 3. The length of the matrix, from proximal to distal along the mid-portion of the great toe is measured.

Variable 4. The length of the nail bed is measured as above from where the colour changes to the end of the bed.

Variable 5. The extensor hallucis tendon is also measured, from lateral to medial, at the DIPJ line.

Variable 6. Also, the thickness of the tendon was measured as well at the DIPJ line

Variable 7. The extensor hallucis tendon was released from the base of the distal phalanx until the proximal nail matrix is reached, and the length of the tendon taking the DIPJ line as the reference until the proximal nail matrix is measured (Fig. 3a,b).

Variable 8. Once the tendon is released until the proximal fold of nail matrix is reached, we continue releasing the tendon underneath of the nail bed until its de-insertion from the dorsal aspect of the distal phalanx of the great toe.

\section{Statistical analysis}

The variables measured were summarized as the mean \pm standard deviations (SD), as well as the 95\% confidence interval (95\% CI), minimum and maximum values, range, and median calculated to describe data distribution. The Kolmogorov-Smirnov testing was used to confirm if there were normal distributions for each measurement. In all of the analyses, statistical significance was established with a $P<0.05$. All the analyses were performed with commercially available software (sPss 19.0, Chicago, IL, USA).

\section{Results}

The results of the measurements are shown in Table 1 .

The variables $1,2,3$ and 8 have a normal distribution and variables 4, 5, 6 and 7 have an abnormal distribution. The proximal edge of the nail matrix was observed in all specimens. The terminal extensor hallucis tendon insertion was clearly identified in all specimens and the main finding was that the extensor hallucis tendon is attached distally along the dorsal aspect to the distal phalanx of the great toe until its end where the extensor hallucis tendon is de-inserted in all specimens. Thus, in all specimens, the extensor hallucis longus tendon reaches the distal part of the distal phalanx covering it completely.

The nail is considered to be a specialized modification of the skin-an 'epidermal appendage'. Namely, the nail is functionally integrated with the musculoskeletal system. The current study analysed how the nail is functionally linked to the distal phalanx and the several distal interphalangeal joint structures, including the extensor tendon fibres and the collateral ligaments. Histological images exhibiting this link are provided (Fig. 4). The extensor tendon, in particular, continues from its bony insertion to envelope the dorsal aspect of the distal phalange, and the collateral ligaments form an integrated network on the sides of the joint, thereby helping to anchor the nail margins. This continuum of connective tissue tendon structure bundles attached along the dorsal aspect to the distal phalanx of the great toe merges with the dorsal aspect on the distal phalanx and with the numerous cutaneous ligaments that anchor the fatty pads of the toe pulp to the skin (Fig. 5).

\section{Discussion}

The most important function of the toe nail is in its proprioceptive role, its protection of the digit, and in the control of the toe pulp. Damage to the matrix can lead to notable disfigurement of the nail plate. ${ }^{18}$ The location of the matrix with respect to the extensor tendon has clinical implications.

Similar studies have been performed in the hand. Numerous studies of fingers have noted the close relationship of the extensor tendon to the matrix, ${ }^{8-14}$ but no studies have examined the exact anatomic distance in the great toe. A study of the surface anatomy of the matrix of the fingernail by Reardon et al. ${ }^{11}$ defined the proximal border of the nail bed with respect to its distance from the distal interphalangeal joint. While these investigators noted that the insertion of the extensor tendon was always more proximal to the most proximal aspect of the matrix, no attempt was made to quantify this distance. One study 
Table 1 Results of the descriptive statistics of the extensor hallucis longus tendon

\begin{tabular}{|c|c|c|c|c|c|c|c|c|}
\hline$\#$ & Variable measurements $(\mathrm{mm}) \mathrm{N}=\mathbf{5 0}$ & Mean \pm SD & 95\% CI Low-Up & Min. & Max. & Range & Median & $P$ value* \\
\hline 1 & Width nail matrix at proximal aspect & $15.63 \pm 1.36$ & $15.24-16.02$ & 12.50 & 19.20 & 6.70 & 15.45 & 0.782 \\
\hline 2 & Width nail matrix at distal nail aspect & $15.51 \pm 1.36$ & $15.12-15.90$ & 12.30 & 19.0 & 6.70 & 15.30 & 0.720 \\
\hline 3 & Length of matrix & $4.39 \pm 1.04$ & $4.09-4.68$ & 2.30 & 6.50 & 4.20 & 4.25 & 0.100 \\
\hline 4 & Length of bed & $15.20 \pm 3.45$ & $14.22-18.19$ & 8.80 & 24.90 & 16.10 & 14.05 & 0.013 \\
\hline 5 & Tendon width & $4.44 \pm 0.67$ & $4.25-4.63$ & 3.40 & 5.50 & 2.10 & 4.60 & 0.001 \\
\hline 6 & Tendon thickness & $1.49 \pm 0.39$ & $1.38-1.60$ & 1.0 & 2.60 & 1.60 & 1.50 & 0.001 \\
\hline 7 & $\begin{array}{l}\text { Distance of tendon from DIPJ line to } \\
\text { proximal nail matrix }\end{array}$ & $7.05 \pm 1.08$ & $6.74-7.36$ & 5.50 & 8.80 & 3.30 & 7.40 & 0.001 \\
\hline 8 & $\begin{array}{l}\text { Releasing the tendon underneath of } \\
\text { the nail bed until its desinsertion }\end{array}$ & $19.59 \pm 3.05$ & $18.72-20.46$ & 13.40 & 27.20 & 13.80 & 19.55 & 0.117 \\
\hline
\end{tabular}

\#, Variable number; mm, millimeters; DIPJ, Distal Interphalangeal joint; Min., minimum; Max., maximum; SD, standard deviation; 95\% Cl, 95\% confidence interval; Low, inferior limit; Up, superior limit.

${ }^{*} P$-values are from Kolmorov-Smirnov-Lilliefords test. $P<0.05$ was considered statistically significant with a confidence interval of $95 \%$.

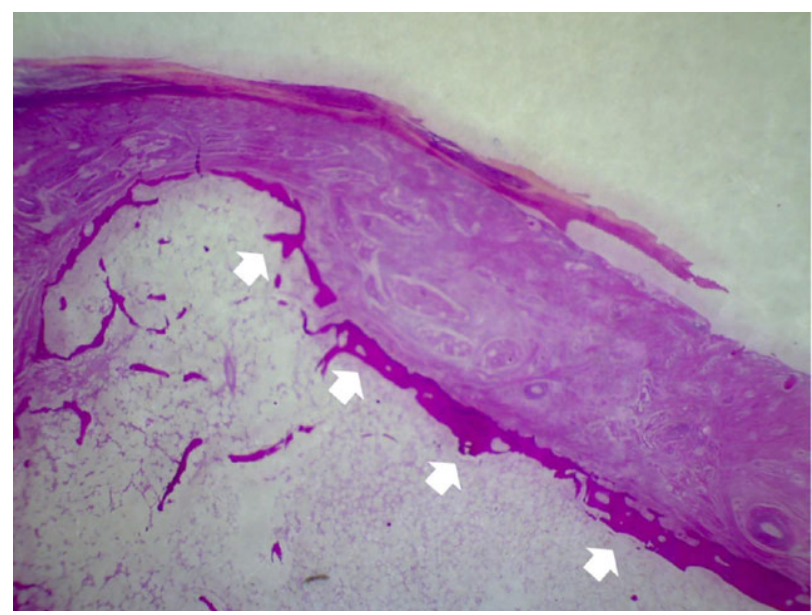

Figure 4 Histology images, tendon bundles attached along the distal phalanx of the hallux (Short arrows) (20x). Haematoxylin and eosin stain.

demonstrated that the proximal limit of the matrix in the human finger is extremely close to the terminal extensor tendon bony insertion and states the average distance from the terminal extensor tendon insertion to the proximal edge of the nail matrix was found to be $1.2 \mathrm{~mm}$ apart. ${ }^{19}$ Others have reported a very small amount of excursion of the digital terminal tendon at the hand that varied between 1 and $2 \mathrm{~mm}$ at the distal interphalangeal joint. ${ }^{17}$

Further, others have stated that the fingernail arises from the matrix which is usually attached to the periosteum of the distal phalanx, and it is this attachment that firmly anchors the nail plate in position ${ }^{20,21}$ and nail bed. A similar study examining the extent of the matrix in toenails reported that the matrix extended to the insertion of the extensor tendon but did not quantify the distance. ${ }^{9}$ Ours is the first study performed on the

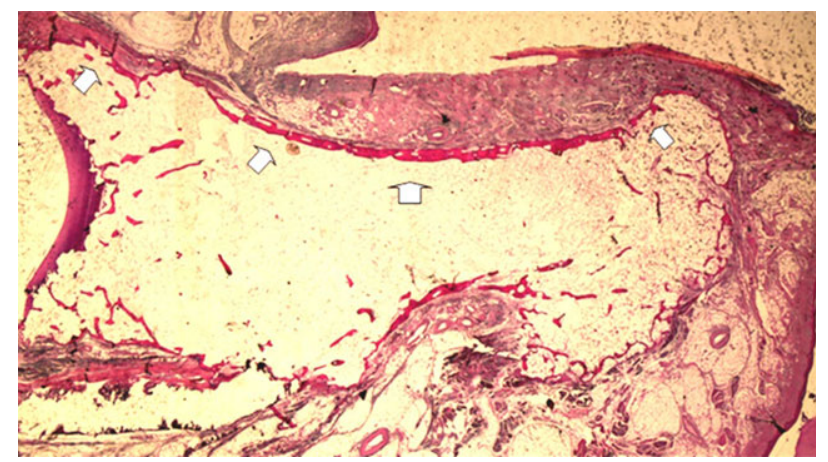

Figure 5 Extensor hallucis tendon continues attached along the dorsal aspect of the distal phalanx of the great toe (Short arrows). Convex and concave structures forming the interphalangeal joint of great toe.

great toe to quantify the distances between the matrix of the nail and the insertion of extensor hallucis longus tendon. In great toes, we have determined that the extensor tendon is between the matrix and the phalanx and extend dorsally to the distal aspect of the distal phalanx. The nail matrix of the great toe is not attached to the periosteum of the dorsal aspect of the base of the distal phalanx as is the case for fingers, because the extensor hallucis tendon is plantar or directly underneath the nail matrix and the tendon is dorsal to the bone. Our anatomic study demonstrates that the proximal limit of the matrix and nail bed in the human great toe are dorsal and overlapping the terminal extensor hallucis longus tendon until its distal bony insertion in all specimens.

Through dissection and histopathologic studies, we have demonstrated that the extensor hallucis longus tendon of the great toe ends in the dorsal area of the distal phalanx of the hallux, in its most distal area. There is a close relationship between the extensor hallucis longus tendon and the matrix of the nail, 
since the nail is functionally linked to the distal area of the distal phalanx and to the structures of the distal interphalangeal joint, including the extensor hallucis longus tendon. These results are significant because they demonstrate that the extensor hallucis longus tendon is inserted into the dorsal and distal area of the distal phalanx, merging with the periosteum, whereas reports in the literature ${ }^{22-25}$ claim that this tendon is attached to the dorsal base area of the distal phalanx. These results are very important, because they show that knowledge of surgical anatomy is essential to prevent intra-operative damage of the nail matrix as well as post-operative complications in nail surgery and osteotripsia (scraping the nail or bone) techniques. Infections at the nail matrix after nail surgery could affect by contiguity the extensor hallucis tendon underneath and spread the infection proximal following the trajectory extensor hallucis tendon affected. Alternatively, the great toe plays an important role in both form and biomechanical function of the foot. In standing, the great toe exerts more pressure than those of the five metatarsal heads and the heel. It also poses a pressure twice that of the other four toes. ${ }^{17}$ During the swing phase of gait, as the great toe is passively dorsiflexed by traction of the concentric contraction of the extensor hallucis longus muscle, we also suggested the possibility that the continuous traction of the extensor hallucis longus, due to its insertion, could be the cause of subungual exostoses and exostoses of mechanical origin.

\section{References}

1 Howard F. Symposium: fingertip injuries. Contemp Orthop 1986; 13: 57-86.

2 Rockwell W, Wray R. Surgery Ofthe Hand and Upper Extremity. In: Peimer C, ed. McGraw-Hill, New York, NY, 1996: 1101-1111.

3 Coyle MP, Leddy JP. Injuries of the distal finger. Prim Care 1980; 7: 245258.

4 Guy RJ. The etiologies and mechanisms of nail bed injuries. Hand Clin 1990; 6: 9-19.

5 De Berker D, Mawhinney B, Sviland L. Quantification of regional matrix nail production. Br J Dermatol 1996; 134: 1083-1086.

6 Zook EG, Van Beek AL, Russell RC, Beatty ME. Anatomy and physiology of the perionychium: a review of the literature and anatomic study. $J$ Hand Surg Am 1980; 5: 528-536.
7 Ditre CM, Howe NR. Surgical anatomy of the nail unit. J Dermatol Surg Oncol 1992; 18: 665-671.

8 Guéro S, Guichard S, Fraitag SR. Ligamentary structure of the base of the nail. Surg Radiol Anat 1994; 16: 47-52.

9 Hyder N. Ingrowing toe nails: the extent of the germinal matrix. J Bone Joint Surg Br 1994; 76: 501-502.

10 Keyser JJ, Littler JW, Eaton RG. Surgical treatment of infections and lesions of the perionychium. Hand Clin 1990; 6: 137-153.

11 Reardon CM, McArthur PA, Survana SK, Brotherston TM. The surface anatomy of the germinal matrix of the nail bed in the finger. J Hand Surg Br 1999; 24: 531-533.

12 Shrewsbury M, Johnson RK. The fascia of the distal phalanx. J Bone Joint Surg Am 1975; 57: 784-788.

13 Zook EG. Complications of the perionychium. Hand Clin 1986; 2: 407427.

14 Zook EG. Anatomy and physiology of the perionychium. Hand Clin 1990; 6: 1-7.

15 Austin RT. A method of excision of the germinal matrix. Proc R Soc Med 1970; 63: 757-758.

16 Brewster NT, Howie CR. Excision of the germinal matrix: a modification. J R Coll Surg Edinb 1995; 40: 59.

17 Schweitzer TP, Rayan GM. The terminal tendon of the digital extensor mechanism: Part I, anatomic study. J Hand Surg Am 2004; 29: 898-902.

18 Baran R, de Berker D, Holzberg M, Thomas L. Baran and Dawber's Diseases of the Nails and their Management, 4th edn. Chichester: WileyBlackwell, 2012.

19 Shum C, Bruno RJ, Ristic S, Rosenwasser MP, Strauch RJ. Examination of the anatomic relationship of the proximal germinal nail matrix to the extensor tendon insertion. J Hand Surg Am 2000; 25: 1114-1117.

20 Tortora G, Anagnostakos N. Principles of Anatomy and Physiology, 6th edn. Harper Collins, New York, NY, 1990.

21 Wright A. Diseases of the Nails in ABC of Dermatology, 2nd edn. BMJ Publishing Group, London, 1994.

22 McCarthy DJ. Anatomic considerations of the human nail. Clin Podiatr Med Surg 2004; 21: 477-491.

23 Wang QC, Johnson BA. Fingertip injuries. Am Fam Physician 2001; 63: 1961-1966.

24 Kelikian A, Sarrafian S. Sarrafian's Anatomy of the Foot and Ankle: Descriptive, Topographic, Functional. Philadelphia: Lippincott Williams \& Wilkins, 2011.

25 Al-saggaf S. Variations in the insertion of the extensor hallucis longus muscle. Folia Morphol (Warsz) 2003; 62: 147-155. 